PROCEEDINGS OF THE

AMERICAN MATHEMATICAL SOCIETY

Volume 134, Number 12, December 2006, Pages 3525-3530

S 0002-9939(06)08378-X

Article electronically published on May 31, 2006

\title{
THE MAXIMUM PRINCIPLE FOR THE BERGMAN SPACE AND THE MÖBIUS PSEUDODISTANCE FOR THE ANNULUS
}

\author{
ALEXANDER SCHUSTER
}

(Communicated by Juha M. Heinonen)

\begin{abstract}
It is shown that the formula for the Möbius pseudodistance for the annulus yields better estimates than previously known for the constant in the Bergman space maximum principle.
\end{abstract}

\section{INTRODUCTION}

The Bergman space $A^{2}$ is the set of functions analytic in the unit disk $\mathbb{D}=\{z \in$ $\mathbb{C}:|z|<1\}$ with

$$
\|f\|=\left\{\frac{1}{\pi} \int_{\mathbb{D}}|f(z)|^{2} d A(z)\right\}^{\frac{1}{2}}<\infty,
$$

where $d A$ denotes Lebesgue area measure. An important result in the theory of Bergman spaces is the so-called Korenblum maximum principle, which we shall also refer to as the Bergman space maximum principle.

Theorem 1. There exists $c \in(0,1)$ with the property that whenever $f$ and $g$ are functions that are analytic in $\mathbb{D}$ and satisfy $|f(z)| \leq|g(z)|$ for $c<|z|<1$, then $\|f\| \leq\|g\|$.

First conjectured by Korenblum [5, the maximum principle was proved by Hayman 2. with $c=0.04$. Hinkkanen [3] later improved upon Hayman's constant by showing that the result holds for $c=0.157 \ldots$ Moreover, he proved that it is valid more generally in the $L^{p}$ Bergman space, where $1 \leq p<\infty$. The value of the best constant, even for $p=2$, remains a mystery. Setting $f(z)=c$ and $g(z)=z$ shows that $c<1 / \sqrt{2}=0.707 \ldots$. Wang [6] has recently shown that $c<0.69472 \ldots$ by considering a certain class of singular inner functions. Our contribution in this paper is to show that the Korenblum maximum principle holds with $c=0.21$.

The paper is organized as follows. In Section 2, much of which follows Hinkkanen [3], we introduce the notation and obtain some preliminary estimates. In Section 3 we discuss the Möbius pseudodistance of the annulus and apply it to obtain the necessary estimates for our proof of the maximum principle. Section 4 consists of the application of our ideas to the setting of the Fock space of entire functions.

Received by the editors September 15, 2004 and, in revised form, June 15, 2005.

2000 Mathematics Subject Classification. Primary 30H05.

Key words and phrases. Bergman space, Fock space, maximum principle.

(C)2006 American Mathematical Society 


\section{Preliminaries}

Let $c \in(0,1)$ be a constant to be determined later, and suppose that $f$ and $g$ are functions analytic in $\mathbb{D}$ satisfying $|f(z)| \leq|g(z)|$ for $z \in A(c, 1)$, where $A\left(r_{1}, r_{2}\right)=$ $\left\{z \in \mathbb{C}: r_{1}<|z|<r_{2}\right\}$. Our goal is to show that

$$
\int_{D(c)}\left(|f(z)|^{2}-|g(z)|^{2}\right) d A(z) \leq \int_{A(c, 1)}\left(|g(z)|^{2}-|f(z)|^{2}\right) d A(z),
$$

where $D(c)$ is the open disk of radius $c$ centered at the origin.

Define the function $\omega=f / g$, which by hypothesis is analytic and satisfies $|\omega| \leq 1$ in $A(c, 1)$. In fact, we may assume, without loss of generality, that $|\omega|<1$ in $A(c, 1)$, since otherwise $|f|=|g|$ in $\mathbb{D}$ and the result holds trivially. We may likewise assume that $f$ is not identically equal to 0 .

For $c<\rho<1$, choose $\zeta_{\rho}$ such that $\left|\zeta_{\rho}\right|=\rho$ and $\left|\omega\left(\zeta_{\rho}\right)\right|=\sup \{|\omega(z)|:|z|=\rho\}$, and define $\omega_{\rho}=\omega\left(\zeta_{\rho}\right)$. We will assume, without loss of generality, that $\zeta_{\rho}=\rho$.

Let $0<r<c<\rho<1$. Following Hinkkanen, we use the inequality $|\alpha|^{2}-|\beta|^{2} \leq$ $2\left|\alpha^{2}-\alpha \beta\right|$ and the fact that, for a subharmonic function $h$ and $0<r_{1}<r_{2}<1$, $\int_{0}^{2 \pi} h\left(r_{1} e^{i \theta}\right) d \theta \leq \int_{0}^{2 \pi} h\left(r_{2} e^{i \theta}\right) d \theta$, to obtain

$$
\begin{aligned}
\int_{0}^{2 \pi}\left(\left|f\left(r e^{i \theta}\right)\right|^{2}\right. & \left.-\left|g\left(r e^{i \theta}\right)\right|^{2}\right) d \theta \leq \int_{0}^{2 \pi}\left(\left|f\left(r e^{i \theta}\right)\right|^{2}-\left|\omega_{\rho} g\left(r e^{i \theta}\right)\right|^{2}\right) d \theta \\
& \leq 2 \int_{0}^{2 \pi}\left|f^{2}\left(r e^{i \theta}\right)-\omega_{\rho} f\left(r e^{i \theta}\right) g\left(r e^{i \theta}\right)\right| d \theta \\
& \leq 2 \int_{0}^{2 \pi}\left|f^{2}\left(\rho e^{i \theta}\right)-\omega_{\rho} f\left(\rho e^{i \theta}\right) g\left(\rho e^{i \theta}\right)\right| d \theta \\
& =2 \int_{0}^{2 \pi}\left|\omega\left(\rho e^{i \theta}\right)\right| \frac{\left|\omega\left(\rho e^{i \theta}\right)-\omega_{\rho}\right|}{1-\left|\omega\left(\rho e^{i \theta}\right)\right|^{2}}\left(\left|g\left(\rho e^{i \theta}\right)\right|^{2}-\left|f\left(\rho e^{i \theta}\right)\right|^{2}\right) d \theta \\
& \leq 2 \gamma(\rho) \int_{0}^{2 \pi}\left(\left|g\left(\rho e^{i \theta}\right)\right|^{2}-\left|f\left(\rho e^{i \theta}\right)\right|^{2}\right) d \theta,
\end{aligned}
$$

where

$$
\gamma(\rho)=\sup \left\{\frac{\left|\omega(z)-\omega_{\rho}\right|}{1-|\omega(z)|^{2}}:|z|=\rho\right\} .
$$

Multiplying both sides of this inequality by $r$ and then integrating from 0 to $c$ with respect to $r$ yields

$$
\int_{D(c)}\left(|f(z)|^{2}-|g(z)|^{2}\right) d A(z) \leq c^{2} \gamma(\rho) \int_{0}^{2 \pi}\left(\left|g\left(\rho e^{i \theta}\right)\right|^{2}-\left|f\left(\rho e^{i \theta}\right)\right|^{2}\right) d \theta .
$$

Multiply both sides of this last inequality by $\rho(\gamma(\rho))^{-1}$ and integrate from $c$ to 1 with respect to $\rho$ to arrive at

$$
\begin{aligned}
\int_{D(c)}\left(|f(z)|^{2}-|g(z)|^{2}\right) d A(z) & \\
& \leq c^{2}\left(\int_{c}^{1} \gamma(\rho)^{-1} \rho d \rho\right)^{-1} \int_{A(c, 1)}\left(|g(z)|^{2}-|f(z)|^{2}\right) d A(z) .
\end{aligned}
$$

It remains to find $c$ such that the quantity in front of the last integral is bounded above by 1 . To achieve this goal we will need to find a suitable bound for $\gamma(\rho)$, which we proceed to do in the next section. 


\section{The MöBius pseudodistance}

To estimate $\gamma(\rho)$ we recall that the pseudohyperbolic distance $d$ between two points $\alpha, \beta \in \mathbb{D}$ is given by the formula

$$
d(\alpha, \beta)=\left|\frac{\alpha-\beta}{1-\bar{\alpha} \beta}\right| .
$$

By the identity

$$
\frac{|\alpha-\beta|}{1-|\alpha|^{2}}=\frac{d(\alpha, \beta)}{\sqrt{1-d^{2}(\alpha, \beta)}} \frac{\sqrt{1-|\beta|^{2}}}{\sqrt{1-|\alpha|^{2}}},
$$

we see that for $|z|=\rho$,

$$
\frac{\left|\omega(z)-\omega_{\rho}\right|}{1-|\omega(z)|^{2}}=\frac{d\left(\omega(z), \omega_{\rho}\right)}{\sqrt{1-d^{2}\left(\omega(z), \omega_{\rho}\right)}} \frac{\sqrt{1-\left|\omega_{\rho}\right|^{2}}}{\sqrt{1-|\omega(z)|^{2}}} \leq \frac{d\left(\omega(z), \omega_{\rho}\right)}{\sqrt{1-d^{2}\left(\omega(z), \omega_{\rho}\right)}} .
$$

It will therefore behoove us to find an estimate for $d\left(\omega(z), \omega_{\rho}\right)$. To do this, we consider the following notion of the distance between two points.

The Möbius pseudodistance for a domain $D \subset \mathbb{C}$ is defined by the equation

$$
c_{D}^{*}(a, z)=\sup \{d(\omega(a), \omega(z)): \omega \in \operatorname{Hol}(D, \mathbb{D})\},
$$

where $a, z \in D$ and $\operatorname{Hol}(A, B)$ denotes the set of analytic functions from $A$ to $B$. (See the book of Jarnicki and Pflug [4 for an excellent survey of this topic.) A basic property of the Möbius pseudodistance is its invariance with respect to biholomorphic maps. Namely, if $\psi: D \rightarrow \Omega$ is a biholomorphism, then

$$
c_{\Omega}^{*}(\psi(a), \psi(z))=c_{D}^{*}(a, z) .
$$

Since the pseudohyperbolic metric is Möbius invariant,

$$
c_{D}^{*}(a, z)=\sup \{|\omega(z)|: \omega \in \operatorname{Hol}(D, \mathbb{D}), \omega(a)=0\} .
$$

It is shown in 1 that for the annulus $P=A\left(\frac{1}{R}, R\right)$ and $\frac{1}{R}<a<R$,

$$
c_{P}^{*}(a, z)=\frac{f\left(\frac{1}{a},-|z|\right)}{R|z|}|f(a, z)|,
$$

where

$$
f(a, z)=\left(1-\frac{z}{a}\right) \prod_{n=1}^{\infty} \frac{\left(1-\frac{z}{a} R^{-4 n}\right)\left(1-\frac{a}{z} R^{-4 n}\right)}{\left(1-a z R^{2-4 n}\right)\left(1-\frac{1}{a z} R^{2-4 n}\right)} .
$$

From the invariance of the Möbius pseudodistance under biholomorphic maps we obtain the formula

$$
\begin{aligned}
c_{A(c, 1)}^{*}( & (\rho, z)=c_{A\left(\sqrt{c}, \frac{1}{\sqrt{c}}\right)}^{*}\left(\frac{\rho}{\sqrt{c}}, \frac{z}{\sqrt{c}}\right) \\
= & \frac{c f\left(\frac{\sqrt{c}}{\rho},-\frac{|z|}{\sqrt{c}}\right)\left|f\left(\frac{\rho}{\sqrt{c}}, \frac{z}{\sqrt{c}}\right)\right|}{|z|}=\frac{c}{|z|}\left(1+\frac{\rho|z|}{c}\right)\left(1-\frac{z}{\rho}\right) \\
& \times\left|\prod_{n=1}^{\infty} \frac{\left(1+\rho|z| c^{2 n-1}\right)\left(1+\frac{1}{\rho|z|} c^{2 n+1}\right)\left(1-\frac{z}{\rho} c^{2 n}\right)\left(1-\frac{\rho}{z} c^{2 n}\right)}{\left(1+\frac{|z|}{\rho} c^{2 n-1}\right)\left(1+\frac{\rho}{|z|} c^{2 n-1}\right)\left(1-\rho z c^{2 n-2}\right)\left(1-\frac{1}{\rho z} c^{2 n}\right)}\right| .
\end{aligned}
$$

In particular,

$$
c_{A(c, 1)}^{*}\left(\rho, \rho e^{i \theta}\right)=\frac{c}{\rho}\left(1+\frac{\rho^{2}}{c}\right) \sqrt{2(1-\cos \theta)} \prod_{n=1}^{\infty} f_{n}(\rho, c) g_{n}(\rho, c, \theta),
$$


where

$$
f_{n}(\rho, c)=\frac{\left(1+\rho^{2} c^{2 n-1}\right)\left(1+\rho^{-2} c^{2 n+1}\right)}{\left(1+c^{2 n-1}\right)^{2}}
$$

and

$$
g_{n}(\rho, c, \theta)=\frac{1-2 c^{2 n} \cos \theta+c^{4 n}}{\sqrt{1-2 \rho^{2} c^{2 n-2} \cos \theta+\rho^{4} c^{4 n-4}} \sqrt{1-2 \rho^{-2} c^{2 n} \cos \theta+\rho^{-4} c^{4 n}}} .
$$

Note that

$$
f_{n}(\rho, c) \leq \frac{1+c^{2 n+1}}{1+c^{2 n-1}}
$$

A calculus argument shows that

$$
g_{n}(\rho, c, \theta) \leq g_{n}(\rho, c, 0)=\frac{\left(1-c^{2 n}\right)^{2}}{\left(1-\rho^{2} c^{2 n-2}\right)\left(1-\rho^{-2} c^{2 n}\right)} \leq \frac{1-c^{2 n}}{1-c^{2 n-2}},
$$

and so

$$
f_{n}(\rho, c) g_{n+1}(\rho, c) \leq \frac{\left(1+c^{2 n+1}\right)\left(1-c^{2 n+2}\right)}{\left(1+c^{2 n-1}\right)\left(1-c^{2 n}\right)},
$$

which is bounded above by 1 for every $n$ if $c<1 / 4$. Therefore,

$$
\prod_{n=6}^{\infty} f_{n}(\rho, c) g_{n}(\rho, c) \leq\left(\frac{1-c^{12}}{1-c^{10}}\right) \prod_{n=6}^{\infty} f_{n}(\rho, c) g_{n+1}(\rho, c) \leq \frac{1-c^{12}}{1-c^{10}} .
$$

On the other hand, a long and extremely tedious calculation shows that

$$
\sqrt{2(1-\cos \theta)} \prod_{n=1}^{5} g_{n}(\rho, c, \theta) \leq 2 \prod_{n=1}^{5} g_{n}(\rho, c, \pi)=2 \prod_{n=1}^{5} \frac{\left(1+c^{2 n}\right)^{2}}{\left(1+\rho^{2} c^{2 n-2}\right)\left(1+\rho^{-2} c^{2 n}\right)} \text {. }
$$

Putting everything together, we have

$$
c_{A(c, 1)}^{*}\left(\rho, \rho e^{i \theta}\right) \leq F(\rho, c),
$$

where

$$
F(\rho, c)=2 \frac{c}{\rho}\left(1+\frac{\rho^{2}}{c}\right)\left(\frac{1-c^{12}}{1-c^{10}}\right) \prod_{n=1}^{5} \frac{\left(1+\rho^{2} c^{2 n-1}\right)\left(1+\rho^{-2} c^{2 n+1}\right)\left(1+c^{2 n}\right)^{2}}{\left(1+c^{2 n-1}\right)^{2}\left(1+\rho^{2} c^{2 n-2}\right)\left(1+\rho^{-2} c^{2 n}\right)} .
$$

Then

$$
\sup _{|z|=\rho} c_{A(c, 1)}^{*}(\rho, z) \leq F(\rho, c),
$$

and so

$$
\gamma(\rho) \leq \sup _{|z|=\rho} \frac{c_{A(c, 1)}^{*}(\rho, z)}{\sqrt{1-\left(c_{A(c, 1)}^{*}(\rho, z)\right)^{2}}} \leq \frac{F(\rho, c)}{\sqrt{1-F^{2}(\rho, c)}} .
$$

We combine this with (2) to obtain

$$
\begin{aligned}
\int_{D(c)}\left(|f(z)|^{2}\right. & \left.-|g(z)|^{2}\right) d A(z) \\
& \leq c^{2}\left(\int_{c}^{1} \frac{\rho \sqrt{1-F^{2}(\rho, c)}}{F(\rho, c)} d \rho\right)^{-1} \int_{A(c, 1)}\left(|g(z)|^{2}-|f(z)|^{2}\right) d A(z) .
\end{aligned}
$$

A calculation involving Mathematica now shows that when $c=0.21$, the quantity in front of the last integral is less than 1 , and so the proof of the result is complete. 


\section{THE FoCK SPACE}

The Fock space $F$ is the set of entire functions with

$$
\|f\|_{F}=\left\{\int_{\mathbb{C}}|f(z)|^{2} e^{-|z|^{2}} d A(z)\right\}^{\frac{1}{2}}<\infty .
$$

We prove the following analogue of the Korenblum maximum principle in this setting.

Theorem 2. There is a positive constant $c$ with the property that whenever $f$ and $g$ are entire functions satisfying $|f(z)| \leq|g(z)|$ for $|z|>c$, then $\|f\|_{F} \leq\|g\|_{F}$.

Note that the proof of the Bergman space maximum principle can be modified to give the result that there is a constant $c$ with the property that whenever $f$ and $g$ are analytic in $\mathbb{D}$ with $|f(z)| \leq|g(z)|$ for $c<|z|<1$, then

$$
\int_{\mathbb{D}}|f(z)|^{2} e^{-|z|^{2}} d A(z) \leq \int_{\mathbb{D}}|g(z)|^{2} e^{-|z|^{2}} d A(z) .
$$

In particular, the maximum principle for the Fock space is a consequence of the maximum principle for the Bergman space. However, the proof of the former is significantly easier than the proof of the latter, and in addition, we obtain a better constant $(c=0.54)$, so we give the proof below.

All of the notation will be exactly the same as in our proof of Theorem 1 . The first part of the argument will be suitably modified to obtain the inequality

$$
\begin{aligned}
\int_{D(c)} & \left(|f(z)|^{2}-|g(z)|^{2}\right) e^{-|z|^{2}} d A(z) \\
& \leq\left(1-e^{-c^{2}}\right)\left(\int_{c}^{\infty} \gamma(\rho)^{-1} \rho d \rho\right)^{-1} \int_{A(c, \infty)}\left(|g(z)|^{2}-|f(z)|^{2}\right) e^{-|z|^{2}} d A(z) .
\end{aligned}
$$

Again, we have

$$
\gamma(\rho) \leq \sup _{|z|=\rho} \frac{c_{A(c, \infty)}^{*}(z, \rho)}{\sqrt{1-\left(c_{A(c, \infty)}^{*}(z, \rho)\right)^{2}}},
$$

but here this quantity is easier to estimate. To this end, we define the function $H: \mathbb{D}^{*} \rightarrow \mathbb{D}$, where $\mathbb{D}^{*}$ is the punctured unit disk, by the equation $H(\eta)=\omega(c / \eta)$. This is analytic in $\mathbb{D}^{*}$, and since $\omega$ is bounded in $A(c, \infty)$, the function $H$ can be extended to be analytic on all of $\mathbb{D}$. By the Schwarz-Pick lemma, we then have for $|z|=\rho$,

$$
c_{A(c, \infty)}^{*}(z, \rho) \leq d\left(\frac{c}{z}, \frac{c}{\zeta_{\rho}}\right) \leq \frac{2 c \rho}{\rho^{2}+c^{2}},
$$

and so

$$
\gamma(\rho) \leq \frac{2 c \rho}{\rho^{2}-c^{2}} .
$$

Therefore,

$$
\begin{aligned}
& \int_{D(c)}\left(|f(z)|^{2}-|g(z)|^{2}\right) e^{-|z|^{2}} d A(z) \\
& \leq 2 c\left(1-e^{-c^{2}}\right)\left(\int_{c}^{\infty} e^{-\rho^{2}}\left(\rho^{2}-c^{2}\right) d \rho\right)^{-1} \int_{A(c, \infty)}\left(|g(z)|^{2}-|f(z)|^{2}\right) e^{-|z|^{2}} d A(z) .
\end{aligned}
$$


A calculation involving Mathematica shows that when $c=0.54$, the quantity in front of the last integral is less than 1 .

\section{ACKNOWLEDGEMENT}

The author is grateful to Dror Varolin for helpful comments and observations.

\section{REFERENCES}

1. R. Courant and D. Hilbert, Methods of Mathematical Physics. Vol. I, Interscience Publishers, Inc., New York, NY, 1953. MR0065391 (16:426a)

2. W. Hayman, On a conjecture of Korenblum, Analysis (Munich) 19 (1999), 195-205. MR 1705360 (2000e:30041)

3. A. Hinkkanen, On a maximum principle on Bergman space, J. Analyse Math 79 (1999), 335-344. MR1749317 (2000m:30033)

4. M. Jarnicki and P. Pflug, Invariant Distances and Metrics in Complex Analysis, de Gruyter Expositions in Mathematics, 9, Walter de Gruyter \& Co., Berlin, 1993. MR 1242120 (94k:32039)

5. B. Korenblum, A maximum principle for the Bergman space, Publ. Mat. 35 (1991), 479-486. MR.1201570 (93j:30018)

6. C. Wang, Refining the constant in a maximum principle for the Bergman space, Proc. Amer. Math. Soc. 132 (2003), 853-855. MR2019965 (2004i:30017)

Department of Mathematics, San Francisco State University, San Francisco, CaliFORNIA 94132

E-mail address: schuster@sfsu.edu 